\title{
A chromosome-scale assembly of the smallest Dothideomycete genome reveals a unique genome compaction mechanism in filamentous fungi
}

\author{
Bo Wang ${ }^{1,2}$, Xiaofei Liang ${ }^{1 *}$, Mark L. Gleason ${ }^{3}$, Tom Hsiang $^{4}$, Rong Zhang ${ }^{1}$ and Guangyu Sun ${ }^{1 *}$
}

\begin{abstract}
Background: The wide variation in the size of fungal genomes is well known, but the reasons for this size variation are less certain. Here, we present a chromosome-scale assembly of ectophytic Peltaster fructicola, a surface-dwelling extremophile, based on long-read DNA sequencing technology, to assess possible mechanisms associated with genome compaction.

Results: At 18.99 million bases (Mb), P. fructicola possesses one of the smallest known genomes sequence among filamentous fungi. The genome is highly compact relative to other fungi, with substantial reductions in repeat content, ribosomal DNA copies, tRNA gene quantity, and intron sizes, as well as intergenic lengths and the size of gene families. Transposons take up just $0.05 \%$ of the entire genome, and no full-length transposon was found. We concluded that reduced genome sizes in filamentous fungi such as $P$. fructicola, Taphrina deformans and Pneumocystis jirovecii occurred through reduction in ribosomal DNA copy number and reduced intron sizes. These dual mechanisms contrast with genome reduction in the yeast fungus Saccharomyces cerevisiae, whose small and compact genome is associated solely with intron loss.
\end{abstract}

Conclusions: Our results reveal a unique genomic compaction architecture of filamentous fungi inhabiting plant surfaces, and broaden the understanding of the mechanisms associated with compaction of fungal genomes.

Keywords: Compact genome, Genome architecture, Ectophytic, Extreme environment fungi, Oxford Nanopore sequencing, Retroelement

\section{Background}

By the early twenty-first century, sequencing of the human genome was complete [1]. The total number of human genes was predicted to be nearly 25,000 [2]. Because the DNA which encoded proteins accounted for only $1.0 \%$ $1.5 \%$ of the total DNA, the human genome was characterized as a $C$-value paradox; that is, not compact [3]. In

\footnotetext{
*Correspondence: xiaofeiliang@nwsuaf.edu.cn; sgy@nwsuaf.edu.cn ${ }^{1}$ State Key Laboratory of Crop Stress Biology in Arid Areas and College of Plant Protection, Northwest A\&F University, Yangling 712100, Shaanxi Province, China

Full list of author information is available at the end of the article
}

contrast, the genome of the pufferfish (Fugu rubripes) is one-eighth the size of the human genome but it has a similar gene repertoire, so it was classified as a compactgenome vertebrate $[4,5]$. In fungi, the yeast Saccharomyces cerevisiae possesses a highly compact genome because of significant intron loss compared to filamentous fungi [6]. The filamentous fungi Pneumocystis spp. and Taphrina deformans, both of the Taphrinomycotina subphylum, were also recognized to have compact genome structures [7-9]. The Pneumocystis genome exhibits substantial reduction of intron size, ribosomal RNA gene copy number

(c) The Author(s). 2020 Open Access This article is licensed under a Creative Commons Attribution 4.0 International License, which permits use, sharing, adaptation, distribution and reproduction in any medium or format, as long as you give appropriate credit to the original author(s) and the source, provide a link to the Creative Commons licence, and indicate if changes were made. The images or other third party material in this article are included in the article's Creative Commons licence, unless indicated otherwise in a credit line to the material. If material is not included in the article's Creative Commons licence and your intended use is not permitted by statutory regulation or exceeds the permitted use, you will need to obtain permission directly from the copyright holder. To view a copy of this licence, visit http://creativecommons.org/licenses/by/4.0/ The Creative Commons Public Domain Dedication waiver (http://creativecommons.org/publicdomain/zero/1.0/) applies to the data made available in this article, unless otherwise stated in a credit line to the data. 
and metabolic pathways [9], whereas $T$. deformans contains few repeated elements and short intron size, specially, just one ribosomal RNA gene copy [8].

The habitats of compact-genome species are usually extreme environments. It is therefore reasonable to hypothesize that streamlining of genome size and function is driven by restrictions imposed by their lifestyles [3]. Fungi in the sooty blotch and flyspeck (SBFS) complex exclusively colonize plant surfaces, which are extreme environments characterized by prolonged desiccation, nutrient limitation, and exposure to solar radiation [10]. Recent research has presented compelling evidence that SBFS fungi underwent profound reductive evolution during the transition from plantpenetrating parasites to plant-surface colonists [11-14].

Fungal genomes are usually smaller than most animal and plant genomes. It was found that fungal genomes were very diverse in nature varies from $8.97 \mathrm{Mb}$ to 177.57 $\mathrm{Mb}$ [15]. The average genome sizes of Ascomycota and Basidiomycota fungi are 36.91 and $46.48 \mathrm{Mb}$ respectively [15]. The class Dothideomycetes, one of the largest groups of fungi with a high level of ecological diversity, had the average genome sizes of $38.92 \mathrm{Mb}$, ranged from the smallest $21.88 \mathrm{Mb}$ in Baudoinia compniacensis to the largest 177.57 Mb in Cenococcum geophilum $[15,16]$.

Our recently published draft nuclear genome of a representative SBFS fungus, Peltaster fructicola was 18.14 $\mathrm{Mb}$, which has the smallest fungal genome known among Dothideomycetes. The genomic analysis of $P$. fructicola revealed several unique features, including a very small repertoire of repetitive elements and very few plant-penetrating genes, such as those involved in plant cell wall degradation, secondary metabolism, secreted peptidases, and effectors, and showed that the gene number reduction made this genome among the smallest in filamentous fungi [12]. In this study, we aim to achieve whole chromosome sequence assemblies for $P$. fructicola genome using Oxford Nanopore long read sequencing technology and to uncover the possible genome compaction mechanism by comparing to other filamentous fungal genomes.

\section{Results}

Chromosome-scale genome sequence assembly

Oxford Nanopore single-molecule sequencing using one flow cell produced $7.71 \mathrm{~Gb}$ of raw sequence data, and average length of passed reads was $19,278 \mathrm{bp}$. After quality and length filtering, the remaining reads provided approximately 406 fold genome coverage. The 369,827 error-corrected reads (N50 length $=26,789 \mathrm{bp}$ ) were assembled using our "assemble and polish pipeline" to give an assembly of 6 unitigs. Five of the six unitigs were completely sequenced from telomere to telomere without gaps (Fig. 1). The additional unitig was the circular mitochondrial genome. The size of the final assembled nuclear-genome was $18.99 \mathrm{Mb}$, with a N50 length of $3.68 \mathrm{Mb}$, which was composed of five chromosomes
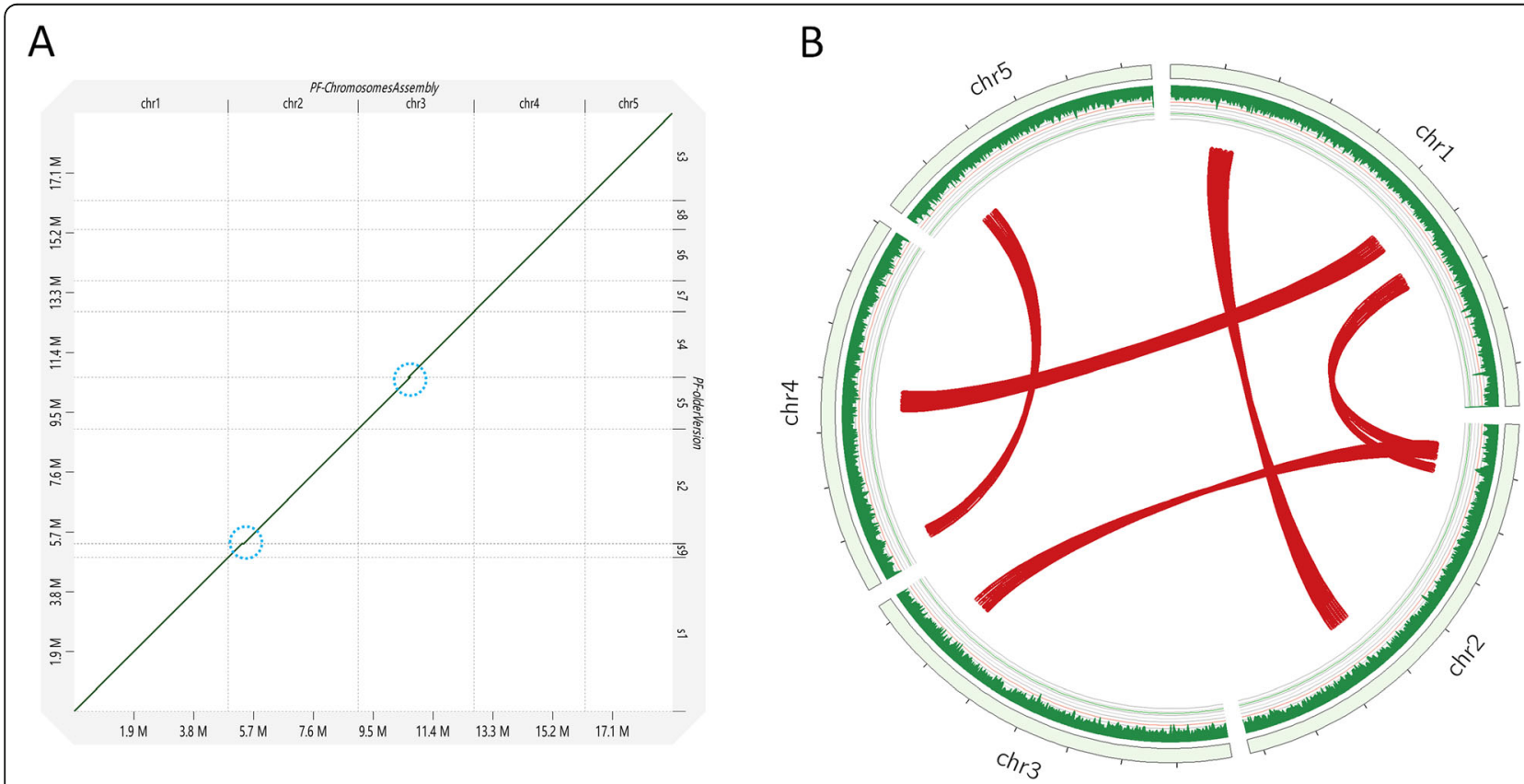

Fig. 1 Chromosome level assembly of $P$. fructicola genome and syntenic blocks of the five chromosomes. a. Dot plot illustrating the comparative analysis of the chromosome level assembly genome and previous draft genome [12]. Scaffolds were grouped into chromosomes. The blue circles highlight major linker regions in chromosome level genome version. b. Circos plot displaying five collinearity blocks among five chromosomes of P. fructicola. From outside to inside, it represents the distribution of chromosome display, GC contents and syntenic regions, respectively 
ranging from $2.77 \mathrm{Mb}$ to $4.89 \mathrm{Mb}$. The five telomere-totelomere chromosomes were categorized as pf_chr1 to pf_chr5, from the largest to the smallest. The genome size was close to the assembly size only using Illumina short-read sequencing $(18.14 \mathrm{Mb})$ and theoretical size $(19.54 \mathrm{Mb})$ [12]. The genome of Peltaster fructicola is smaller than the extremophilic sooty mold Baudoinia compniacensis $(21.88 \mathrm{Mb})$ (Fig. 2a-b), which was the smallest previously reported genome for a filamentous fungus in Dothideomycetes [16].

A relatively small number of protein-coding genes was annotated in P. fructicola (8072) (average size $=500$ aa) (Fig. S1), compared with the fungal phytopathogens Sphaerulina populicola (9739) and Passalora fulva (14, 127). P. fructicola has higher gene density than other characterized Dothideomycetes species, except for $B$. compniacensis (Fig. 3). The genomic size of P. fructicola is similar to that of the basidiomycete Ustilago maydis (19.66 Mb) [17], but P. fructicola has higher gene density (425 per Mb vs. 345 per $\mathrm{Mb}$ ) and shorter average intron (Fig. 2c and Fig. S2) and intergenic length (Fig. 2d). There is little difference in gene density between $P$. fructicola and compact fungal genome of Pneumocystis jirovecii [9] (425 per $\mathrm{Mb}$ vs. 448 per $\mathrm{Mb}$ ), or with fungus Taphrina deformans (431 per $\mathrm{Mb}$ ), but exceeded most of fungi examined (Fig. 3).

Of the 8072 gene models for $P$. fructicola, 8057 were supported by at least one FPKM (Fragments per kilobase of exon per million reads mapped), and 7658 models were supported by at least 10 FPKM. Among the predicted genes, 6010 genes had matches to entries in the PFAM database, 7575 genes had matches in the non-redundant database and 5723 were mapped to Gene Ontology (GO) terms (Fig. S3). We re-predicted a previous draft genome of $P$. fructicola [12] using the pipeline developed in this study (see methods section) and obtained 7604 gene models. To compare gene content between the current and former annotations of $P$. fructicola, we used BUSCO v.1.2 to search for a set of 1438 fungi universal single-copy orthologous genes (FUSCOGs). Among 1438 FUSCOGs, the proportion classified as 'fragmented' declined from $5.8 \%$ in the previous annotation to $3.8 \%$ in the current annotation, and the proportion classified as 'missing' declined from 1.8 to $1.1 \%$. Some fragmented and missing regions were recovered in this new assembly version (Fig. 1a). The BUSCO identification of nearly all (99\%) core fungal genes of the current annotation of $P$. fructicola suggested a high-quality assembled genome and predicted gene set.

\section{Telomere repeat}

Chromosome-scale assembly suggested that the repeat unit in P. fructicola telomeres was TAGGG. This unit was has not been previously reported from other fungi (Telomerase Database: http://telomerase.asu.edu/sequences telomere.html), but was reported from the unicellular heterotrophic flagellate Giardia intestinalis [18], a unicellular heterotrophic flagellate, whose genome is compact [19]. A repeat unit of telomeres of P. fructicola and Giardia spp., formed by five bases, is the shortest compared to all other eukaryote species reported (6-26 bases) (Telomerase Database: http://telomerase.asu.edu/sequences_telomere. html). Interestingly, none of the subtelomeric regions (up to $25 \mathrm{~kb}$ ) in the $P$. fructicola nuclear genome showed homology to each other ( $e$-value $=1 \mathrm{e}-3$, coverage $>10 \%)$. This situation is different from that of Saccharomyces cerevisiae, in which all chromosomal ends contain core $\mathrm{X}$

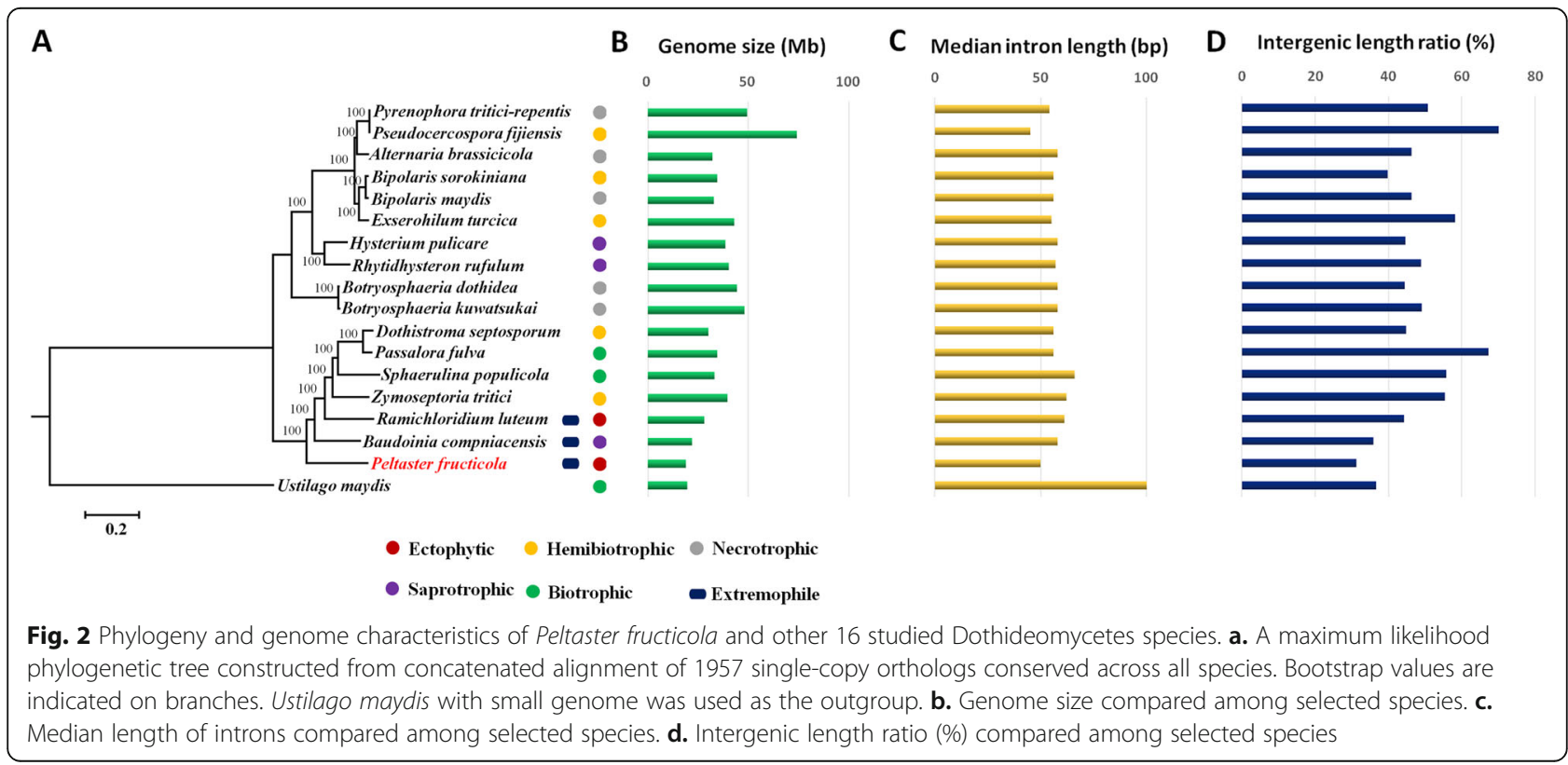




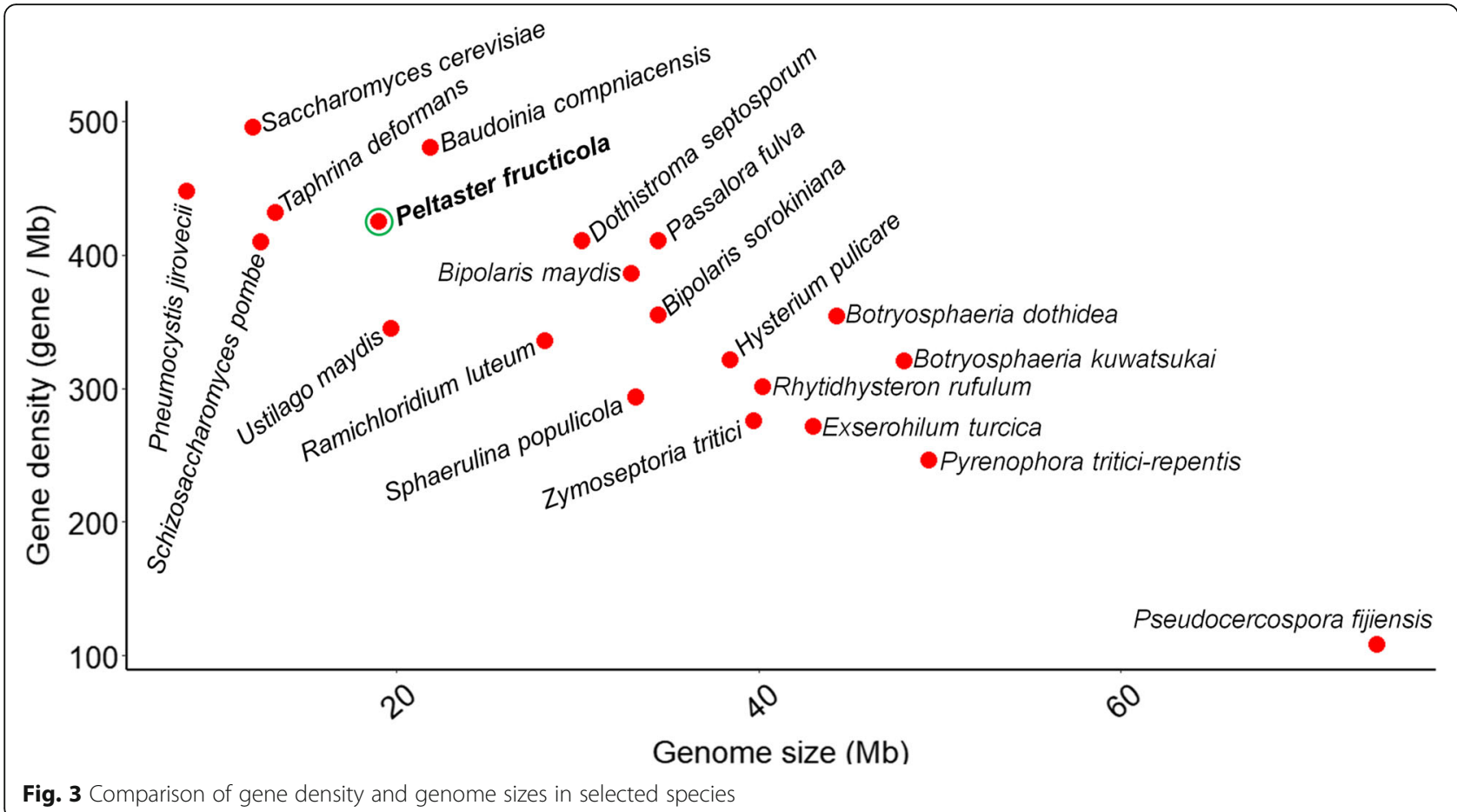

elements [20]. In addition, all subtelomeric regions in $P$. fructicola were of low gene density with only 30 genes detected in the 10 subtelomeric regions composed of $250 \mathrm{~kb}$ (Table S1) resulting in 0.12 genes per kb. In contrast, the average whole genome gene density was 0.425 genes per $\mathrm{kb}$. There was no regularity in the distribution of genes in the subtelomeric region on chromosomes, and most of the genes had unknown functions. The pf_chr2 right arm contained a GH31 gene and pf_chr5 right arm contained an amino acid permease (Table S1). The function of the GH31 gene was predicted to be alpha-glucosidase activity, which can release glucose from the non-reductive end of oligosaccharide substrates by cutting alpha-1,4-glycoside bonds [21]. Amino acid permease is a membrane protein with 12 transmembrane domains whose function is to transport amino acids into cells. Using Phobius software (http://phobius.binf.ku.dk/), we predicted that the g6510.t1 gene had 12 transmembrane domains, which further confirmed that the gene was an amino acid permease.

\section{Decreased chromosome number and relative independence of five chromosomes}

The finished genome of $P$. fructicola contained five chromosomes, much fewer than the 21 chromosomes of its closely related plant-penetrating species, Zymoseptoria tritici [22]. We found that the P. fructicola genome was overall gene-dense with shorter intron (Fig. 2c) and intergenic lengths (Fig. 4a) but longer exon lengths compared to $Z$. tritici genome (exon size median: 328 vs. 300 ) which shows overall gene-sparse (Fig. 4b). Pairwise sequence comparison of the genomes of P. fructicola with $Z$. tritici (Fig. 4b) revealed a high degree of micro-mesosynteny (genome segments having a similar gene content but shuffled order and orientation), likely due to intrachromosomal rearrangements [23]; this level of rearrangement appears to be among the most striking between closely related genera anywhere in the Dothideomycetes [24]. There were no syntenic regions observed between the $P$. fructicola chromosomes and the eight accessory chromosomes of $Z$. tritici (Fig. 4b). Chromosomal fusion may have led to depletion in numbers of $P$. fructicola chromosomes. For example, fusional DNA may have carried a gene that was beneficial to the recipient species, and thus the chromosome (or a large section) carrying this gene may have been retained while sections not essential for environmental adaptation were lost; these processes may help to explain both P. fructicola's massive loss of pathogenicity-related genes and its retention of cutinase and secreted lipases [12, 24].

The pf_chr5 had greater density than the other four chromosomes, whereas rDNA repeat units gave pf_chr2 the lowest gene density. Only 69 collinear genes $(0.85 \%$ of all genes) were detected, in five collinearity blocks (Fig. 1b). One pair of collinear genes located on pf_chr1 and pf_chr2 were involved in DNA repair (Table S2).

\section{Very low repeat content}

Multivariate repeated DNA sequences may account for variations in genome size [25]. Analysis of the repeat content of the chromosome-scale assembly of $P$. fructicola 

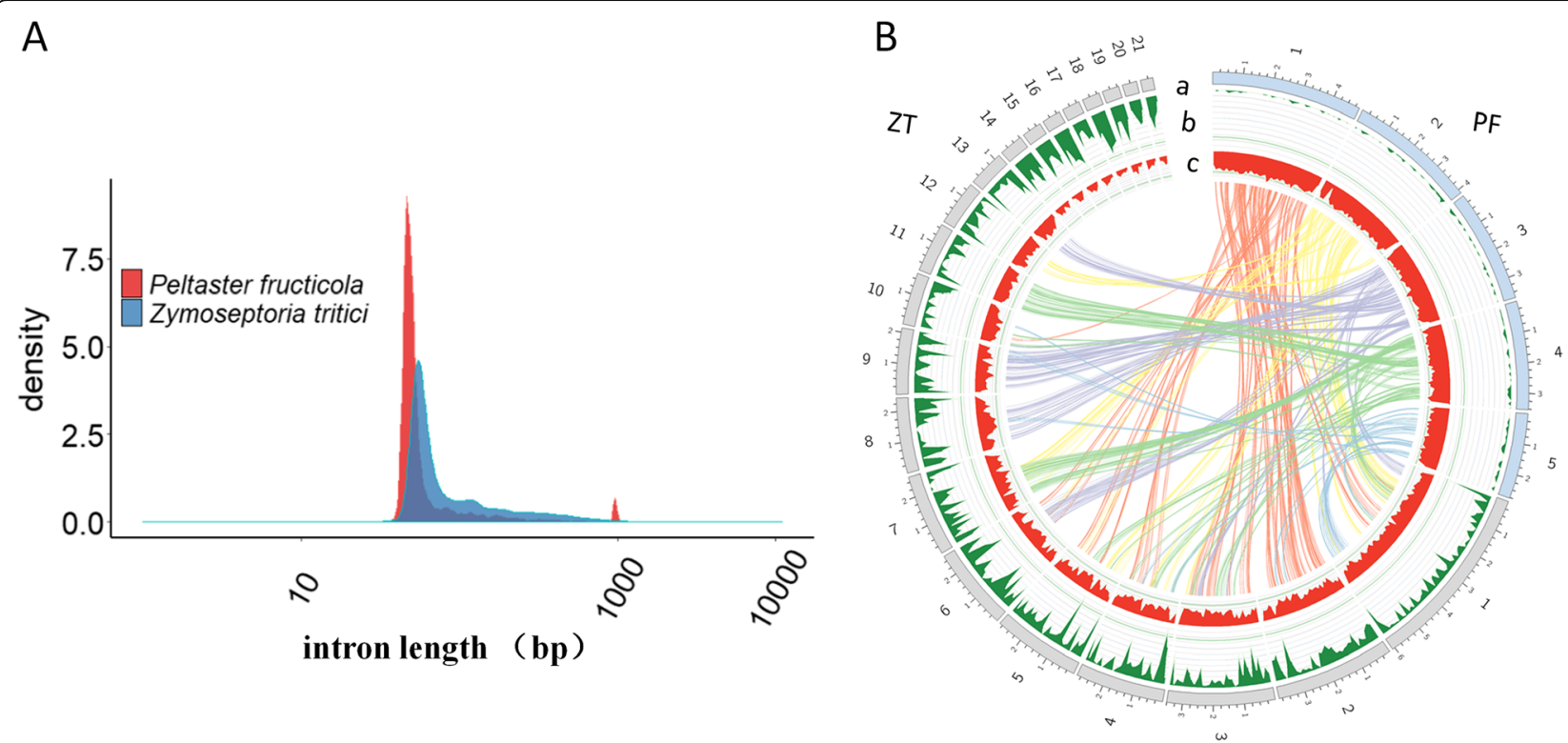

Fig. 4 Length of intergenic region, colinearity, transposable elements (TEs) and gene density analysis between Peltaster fructicola and Zymoseptoria tritici. a. Intergenic length density plot of $P$. fructicola genome and $Z$. tritici genome. b. Syntenic blocks between two species are shown in various color lines (BLASTN coverage $>1 \mathrm{~kb}$ ). P. fructicola (PF) chromosomes are shown as light blue colour, Z. tritici (ZT) 21 chromosomes [22] are shown as colour. Track a-c are the distribution of chromosomes, TEs density and gene density respectively, with densities calculated in $100 \mathrm{~kb}$ windows

revealed that repeat elements comprised only $0.34 \%$. When compared to other highly compact fungi, the repeat content of P. fructicola was also the lowest (Table 2). Most of the repeat elements identified were found in simple repeat sequences $(0.278 \%)$ (Table S3). Only $0.05 \%$ of the genome assembly was classified as transposable element (TE) insertions. A total of $112 \mathrm{TE}$ insertion locations were of multiple origins, representing $11 \mathrm{TE}$ families from the two main TE orders (Class I/retrotransposons and Class II/DNA transposons). Most of the TE insertions were from retroelements $(86.6 \%)$, which were created based on the three primary ingredients: Ty1/Copia long terminal repeats (LTR) elements, Gypsy/DIRS1 LTR elements and Tad1 long interspersed nuclear elements (Table 1). The Gypsy and Copia superfamilies were the main LTRretrotransposon elements (Table 1). Maximum length percentage of total TE length were only 27\% (According to RepBaseEdition-20,170,127) (Fig. 5a), so no full-length TE was detected in the P. fructicola genome (Fig. 5b), and the lengths were very short (Table S3). A total of nine Class II TE families [i.e., 3 hobo-Activator, 1 Helitron, 1 TcMar-Sagan, 1 TcMar-Pogo, 2 TcMar-Fot1, 1 En-Spm, 4 Harbinger, 1 P-element and one unclassified element] were identified. The fragment length of DNA transposons was only $17 \%$ of full length extracted from RepBaseEdition-20, 170,127 (https://www.girinst.org/). The pf_chr4 had the most TE elements $(n=31)$ compared to pf_chr1 $(n=22)$, pf_chr2 $(n=21)$, pf_chr3 $(n=24)$, and pf_chr5 $(n=15)$. The number of DNA transposons was similar to that of $S$. cerevisiae but the number of retroelements was significantly lower (Table 1). When compared to TE families in $Z$. tritici, we found that $P$. fructicola had a reduced battery of Class I and Class II transposable elements (Table 1).

\section{Reduced rDNA and tRNA genes}

Because of the strong positive relationship between rDNA copy number and genome size [26], we examined rDNA copy number to determine its relationship to the small genome size of $P$. fructicola. The $P$. fructicola rDNA unit was defined according to the complete rDNA sequence of Neurospora crassa (GenBank accession: FJ360521) using BLASTN. We obtained a 5932 bp rDNA unit including 18S-5.8S-28S ribosomal genes, which were located on pf_chr2. Like most eukaryotic species [27], 5S rDNA genes of $P$. fructicola were found outside the rDNA units, and were situated on pf_chr1, 3, 4 and 5 . We estimated nine copies of the rDNA gene cassette in P. fructicola according to a computational method using whole-genome short-read DNA sequencing [28]. This copy number was strikingly smaller than that of Saccharomyces cerevisiae $(\sim 560)$ but similar to other filamentous fungi with compact architecture (Table 2), as well as most bacteria [29]. In P. fructicola, 44 tRNA genes were identified by tRNAScan-SE, similar to the total in Pneumocystis jirovecii (71tRNAs) and other Pneumocystis spp.(45 to 47 tRNAs) (9) but much less than that in S. cerevisiae or T. deformans (Table 2), or other eukaryotes (170-570 copies) [30]. 
Table 1 Classified repeat contents in Peltaster fructicola, Saccharomyces cerevisiae and Zymoseptoria tritici. All annotation data were analysis using the pipeline described in method section

\begin{tabular}{|c|c|c|c|}
\hline & P. fructicola & S. cerevisiae & Z. tritici \\
\hline Genome assembly source & Current study & NCBI R64 & NCBI IPO323 \\
\hline Retroelements & 97 & 562 & 2261 \\
\hline LINEs (long interspersed nuclear elements): & 5 & 4 & 537 \\
\hline Penelope & 0 & 0 & 1 \\
\hline Tad1 & 5 & 3 & 376 \\
\hline RTE/Bov-B & 0 & 0 & 97 \\
\hline L1/CIN4 & 0 & 0 & 63 \\
\hline CRE & 0 & 1 & 0 \\
\hline LTR (long terminal repeats) elements: & 92 & 558 & 1376 \\
\hline BEL/Pao & 0 & 0 & 37 \\
\hline Ty1/Copia & 34 & 477 & 347 \\
\hline Gypsy/DIRS1 & 57 & 81 & 991 \\
\hline Ngaro & 0 & 0 & 1 \\
\hline Retroposon (Unclassified) & 1 & 0 & 348 \\
\hline DNA transposons & 15 & 18 & 564 \\
\hline hobo-Activator & 3 & 0 & 85 \\
\hline hAT-hATw & 1 & 0 & 0 \\
\hline hAT-Ac & 2 & 0 & 1 \\
\hline hAT-Restless & 0 & 0 & 84 \\
\hline Helitron & 1 & 1 & 1 \\
\hline TcMar-Sagan & 1 & 0 & 1 \\
\hline TcMar-Pogo & 1 & 0 & 0 \\
\hline TcMar-Fot1 & 2 & 1 & 88 \\
\hline TcMar-Tc1 & 0 & 0 & 40 \\
\hline TcMar-Ant1 & 0 & 1 & 63 \\
\hline Novosib & 0 & 0 & 30 \\
\hline Dada & 0 & 0 & 1 \\
\hline En-Spm & 1 & 7 & 2 \\
\hline DNA (Unclassified) & 1 & 4 & 44 \\
\hline MuLE-MuDR & 0 & 0 & 63 \\
\hline Tourist/Harbinger & 4 & 3 & 61 \\
\hline Other (Mirage, P-element, Transib) & 1 & 1 & 0 \\
\hline Simple repeats: & 1279 & 2828 & 6392 \\
\hline Low complexity: & 78 & 530 & 830 \\
\hline Transposon content (\%) & 0.05 & 3.41 & 10.42 \\
\hline Repeat content (\%) & 0.34 & 5.13 & 12.26 \\
\hline
\end{tabular}

\section{Reduced length of non-coding DNA}

The median intron size of $P$. fructicola was $50 \mathrm{bp}$, only slightly more than that of Pneumocystis jirovecii (45 bp) and Pseudocercospora fijiensis (45 bp), but much less than the median of $S$. cerevisiae (111 bp) [31] and Dothideomycetes species in general $($ median $=57 \mathrm{bp}$, significantly different from $P$. fructicola) (Fig. 2). Intron size distribution in $P$. fructicola compared with others showed that the length of introns tended to be the shortest (Fig. S2). The longest intron size of $P$. fructicola was only $1053 \mathrm{bp}$, much short than others (1356 bp to $42,135 \mathrm{bp}$ ). Intron number of $P$. fructicola 


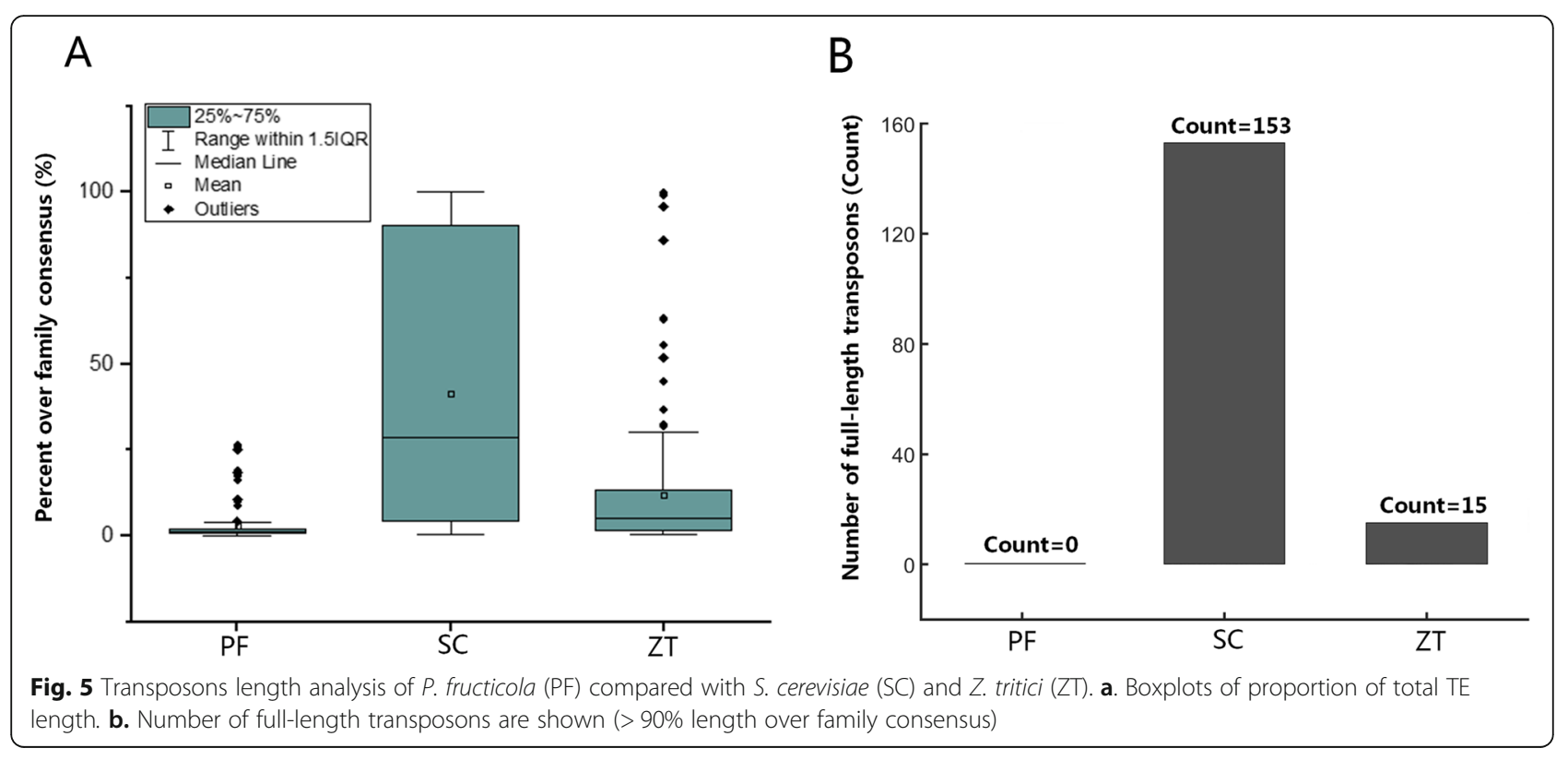

was significant higher than that of S. cerevisiae (Table 2), but the intron sizes in P. fructicola were strikingly smaller compared to $S$. cerevisiae. Intron size has been correlated to TE number [32], and as expected, P. fructicola also had a correlation between small intron size and fewer TEs.

Intergenic regions in P. fructicola occupied only 31\% of the genome (Table 2), which was similar to that of $S$. cerevisiae (26\%), Pneumocystis jirovecii (29\%), and Taphrina deformans (36\%), but smaller than for other Dothideomycetes species (range from $36 \%$ in B. compniacensis to70\% in Pseudocercospora fijiensis). Moreover, $92.2 \%$ of the $P$. fructicola genome was covered by primary transcripts across all five stages. Genome-wide coverage of transcribed regions of the $P$. fructicola genome was significantly higher than for many non-compact fungal species, such as Colletotrichum fructicola (52.4\%), Passalora fulva (60.4\%), Zymoseptoria tritici (70.6\%), Alternaria brassicicola (82\%) and Ustilago maydis (84.0\%), and even exceeded transcribed coverage for the human genome $(\sim 75 \%)$ [2]. Another SBFS fungus, Ramichloridium luteum, which shared some common features with $P$. fructicola, also had a high transcribed coverage (87.3\%).

Table 2 Peltaster fructicola nuclear genome statistics and comparison to other fungal species with highly compact genome

\begin{tabular}{|c|c|c|c|c|}
\hline Species & Peltaster fructicola & Taphrina deformans & Pneumocystis jirovecii & Saccharomyces cerevisiae \\
\hline Chromosomes (count) & 5 & $N A^{a}$ & $N A^{a}$ & 16 \\
\hline Genome size (Mb) & 18.99 & 13.3 & 8.1 & 12.07 \\
\hline GC content (\%) & 51.95 & 49.5 & 29.1 & 38.3 \\
\hline Protein coding genes (count) & 8072 & 5735 & 3898 & 6002 \\
\hline Exons per gene (count) & 2.32 & 2.1 & 3.7 & 1.13 \\
\hline Introns per gene (count) & 1.36 & $N A^{a}$ & $4.7^{\mathrm{b}}$ & 0.06 \\
\hline tRNA genes (count) & 44 & 169 & 71 & 275 \\
\hline rDNA copies (count) & 9 & 1 & 5 & $\sim 560^{b}$ \\
\hline Intergenic distance (median, bp) & 463 & $N A^{a}$ & 326 & 350 \\
\hline Intergenic regions (\%) & 31 & 36 & 29 & 26 \\
\hline Intron distance (median, bp) & 50 & $N A^{a}$ & 45 & 111 \\
\hline Telomere repeat unit & TAGGG & TTAGGG & TTAGGG & $\mathrm{T}(\mathrm{G})_{2-3}(\mathrm{TG})_{1-6}$ \\
\hline Repeat content (\%) & 0.34 & 1.5 & 9.8 & 5.13 \\
\hline Data source & Current study & From reference [8] & From reference [8] & $\begin{array}{l}\text { Analysis in this study. NCBI R64 } \\
\text { genome version was used }\end{array}$ \\
\hline
\end{tabular}

${ }^{a} \mathrm{NA}$, not applicable or not available from the website

${ }^{\mathrm{b}}$ These data are obtained from reference [9] 


\section{Substantial reduction of the size of gene families but retention of all primary metabolic pathways}

Based on a maximum likelihood tree constructed from concatenated alignment of 1957 single-copy orthologs conserved across 18 selected species, $P$. fructicola was clustered with other members from Capnodiales (Fig. 2a). We compared the P. fructicola genome with that of three other Capnodiales species with various lifestyles including the biotrophic plant pathogen Passalora fulva, hemibiotrophic plant pathogen $Z$. tritici, and saprotrophic fungus $B$. compniacensis. In an orthoMCL comparison between the four species, potential clusters of orthologous genes for comparative analyses were determined. A total of 139 genes (59 clusters) were unique to $P$. fructicola (orphans), and 5479 one-to-one orthologous genes (5337 clusters) of $P$. fructicola were identified. About $63 \%$ of the orphans were hypothetical protein-coding or had no homology to sequences in GenBank. Five orphans containing lipase_GDSL_2 domain (PF13472), a family of presumed lipases, were probably associated with colonization of the epicuticular wax layer on plant surfaces. $P$. fructicola had reduced cluster size; that is, it and $B$. compniacensis had the minimum number of multi-gene clusters ( 2 genes at least), compared with Z. tritici, Passalora fulva or Saccharomyces cerevisiae (Fig. S4). Some genes involved in transmembrane transport and hydrolase glycosyl chain activity were multi-gene clusters in $Z$. tritici, but there was only one copy of each cluster in P. fructicola (Table S4). Reduction in the size of gene families may be a key contributor to $P$. fructicola's exceptionally diminutive genome among Dothideomycetes fungi. Although reduction in the number of gene families often occurred in P. fructicola [12], key metabolic pathways were completely retained, including genes involved in carbohydrate metabolism, amino acid metabolism, nucleotide metabolism, lipid metabolism and cofactor metabolism (Table S5, Table S6, Table S7, Table S8 and Table S9).

\section{Discussion}

Sooty blotch and flyspeck (SBFS) fungi occupy an exclusively surface-dwelling niche. An ecologically distinctive group of plant pathogens, they have smaller genomes than their plant-penetrating parasite relatives [12-14]. The SBFS fungus Peltaster fructicola was found to possess the smallest known genome size in Dothideomycetes [15], which is one of the largest groups of fungi with a high level of ecological diversity and life styles [15]. Expect, $P$. fructicola has higher gene density than other characterized Dothideomycetes species, only except for B. compniacensis. Comparing to fungi out of Dothideomycetes, $P$. fructicola also had similar gene density with highly compact fungal species Pneumocystis jirovecii and Taphrina deformans $[8,9]$. Furthermore, by analysis of genome architecture, we confirmed that P. fructicola is not only the smallest but also the most compact fungus in Dothideomycetes, and highlighted the mechanisms associated with formation of its compact genome structure.

A remarkable feature of $P$. fructicola was a reduction in the number of repeats, a similar mechanism to that reported for the Ascomycota fungus Pneumocystis jirovecii [9], the basidiomycete yeast Mixia osmundae [33], the early-diverging fungus Encephalitozoon cuniculi [34], the algae Ostreococcus tauri and Cyanidioschyzon merolae $[3,35]$, the bladderwort plant (Utricularia gibba) [36], the pufferfish (Tetraodon nigroviridis) [37], and the Antarctic midge Belgica antarctica [38], whose small genomes are also characterized by compactness. An approximately $0.3 \%$ repeat content of the assembled $P$. fructicola genome was lower than that of $B$. compniacensis $(0.8 \%)$ which was formerly the smallest fraction reported in Dothideomycetes [15]. Now, repeat content of $P$. fructicola was the smallest proportion reported not only in Dothideomycetes but also in the above eukaryotic compact species. Transposable elements (TEs) are enigmatic genetic units that play important roles in the evolution of eukaryotic and prokaryotic genomes [39]. Fungal genomes have varied TEs contents (from $0.7 \%$ of Botrytis cinerea to $70 \%$ of Blumeria graminis f. sp. hordei) [39]. The 0.05\% TE composition of $P$. fructicola shows substantial reduction. In particular, the number of retroelements was significantly fewer than that of the highly compact fungus $S$. cerevisiae. Avian malaria parasites Plasmodium falciparum, $P$. knowlesi and P. relictum are the only eukaryotes having no full-length TE detected in their genomes [40]. No full-length TE was detected in the $P$. fructicola genome, and all LTRs were fragments that entailed loss of all retroelementinternal sequences. $P$. fructicola may be the first fungus reported that contains no complete TEs. LTRs do not always lead to genome size expansion over evolutionary time, because there are also processes for rapid removal of DNA, such as from flowering plant genomes by accumulated deletions caused by illegitimate recombination [41-43]. Such recombination may also play an important role in genome shrinkage of fungal species.

Streamlining theory predicts that oligotrophic bacteria $[30,44]$ should have smaller genome sizes, with few rDNA copies, than non-oligotrophic bacteria. We found that the oligotrophic fungus $P$. fructicola followed this trend, containing just nine rDNA copies. It was generally assumed that rDNA redundancy allows the cell to maintain a functional ribosome in diverse environments and that a higher rDNA copy number allows for an increased rate of rRNA synthesis, resulting in a higher level of ribosome production and more rapid growth [45]. The reduction to nine rDNA copies in $P$. fructicola may reflect its genome stability as a result of adaptation to an extreme environment [46]. Few rDNA copies together with a minimal number of tRNA genes, suggest slow 
transcription and translation processes in P. fructicola, but growth efficiency could be high [29]. Interestingly, small and compact genomes of Taphrina deformans, Pneumocystis jirovecii, Ostreococcus tauri and Cyanidioschyzon merolae were achieved by this feature, with 1-5 rDNA copies, resulting in a strong positive relationship between rDNA copy number and genome size [25] or compact architecture. In the present study, the chromosome-scale assembly confirmed that TAGGG was the telomere repeat sequence in all $P$. fructicola chromosomal ends. In most filamentous fungi, the telomeres are composed of many copies of the sequence TTAGGG [47], and the P. fructicola telomere sequence, TAGGG, had never been found previously in fungal species. Most telomeric repeats range from 6 to $26 \mathrm{nu}-$ cleotides [47], but the telomere repeat sequence of $P$. fructicola is composed of only 5 nucleotides, the shortest known telomere repeat unit among fungi.

Intron size has been positively correlated to TE number [32, 38], suggesting that small intron size of $P$. fructicola may be a result of fewer TEs. The intron size of $P$. fructicola was much less than that of a variety of genome sequenced Dothideomycetes species and the highly compact genome yeast $S$. cerevisiae. When compared to species outside the fungal kingdom, mean length of introns was much less than for the smallest free-living eukaryote, Ostreococcus tauri (103 bp) [3]. We suggest that reduction of intron size of the oligotrophic fungus $P$. fructicola might save a large amount of energy and resources used for intron processing. Moreover, intergenic regions in P. fructicola were the shortest among Dothideomycetes species, and the occupied proportion of the genome was close to that of $S$. cerevisiae and the compact yeast-like fungus $P$. jirovecii [9]. Shortening of intergenic regions and reducing intron size were clearly two major mechanisms behind the intense degree of genome compaction of $P$. fructicola. By transcribed coverage analysis of $P$. fructicola, Colletotrichum fructicola, Passalora fulva, Zymoseptoria tritici, Alternaria brassicicola, and Ustilago maydis, we suggest that its small introns and high relative contents of exon information may lead to a relatively high level of transcriptional coverage compared to these none compact fungal species examined. In the $S$. cerevisiae genome, loss of intron number was a major compaction mechanism. Therefore, we suggest that reduction of intron length rather than intron number in P. fructicola, Taphrina deformans and Pneumocystis jirovecii genome is the compaction mechanism. This is highly unusual in filamentous fungi.

Range of chromosome numbers in Ascomycota was 4 in Fusarium graminearum to 21 in Zymoseptoria tritici $[21,48]$. Five chromosomes of the finished P. fructicola genome significantly reduced compared to 21 chromosomes in its related species $Z$. tritici. Compared to other species whose genomes are compact, a reduced number of chromosomes is a unique feature of $P$. fructicola, for example, 11 chromosomes in Encephalitozoon cuniculi, 16 in $S$. cerevisiae, and 20 in O. tauri and C. merolae [3, 34, 35]. Gene function of the five $P$. fructicola chromosomes was almost entirely non-redundant and only five collinearity blocks were detected. Cluster analysis of gene families revealed that $P$. fructicola had reduction in the size of gene families compared to non-compact genomes. Although genes involved in plant cell wall degradation, secreted peptidases and effectors were drastically reduced because the fungus does not interact with host immune defenses [12], all other major metabolic pathways were basically retained. It was different from the genome compact species Pneumocystis jirovecii, with substantial reductions of many metabolic pathways [9]. So we suggest that high gene density of $P$. fructicola appeared to constrain genome architecture, rather than gene content. Nevertheless, $P$. fructicola is an interesting anomaly among sequenced filamentous fungi in having a strikingly compact genome.

\section{Conclusion}

In this study, we report the nuclear-genome sequence of Peltaster fructicola in a chromosome-scale assembly. Analysis of genome architecture and gene content revealed that $P$. fructicola genome possesses the smallest and the most compact genome in Dothideomycetes, and further yielded new insights into mechanisms for compaction in filamentous fungi. The intense degree of genome compaction in P. fructicola appeared to be the result of several processes. One major factor is the very low repeat content. Shortening of intergenic regions, small introns and very high coverage of transcribed regions are additional important factors. A reduced number of chromosomes and diminutive size of gene families also played roles in the intense genome compaction. Compaction mechanisms of filamentous fungus such as P. fructicola, Taphrina deformans and Pneumocystis jirovecii are distinct from those of yeasts such as Saccharomyces cerevisiae. Shortening of intergenic regions and reduction of intron size were clearly two major factors in the exceptional degree of genome compaction of $P$. fructicola. We considered that $P$. fructicola genome was highly compact by means of mechanisms that are distinct from those of S. cerevisiae. Interestingly, most of these genomic features of $P$. fructicola are shared by the extremophilic filamentous saprophyte $B$. compniacensis and another SBFS fungus, Ramichloridium luteum. Even some animals and plants that live in extreme environments exhibit genome reduction, and several kingdoms may share similarities in mechanisms of genome compact in such challenging habitats. Characterizing the exceptionally tiny genome of $P$. fructicola substantially broadens our understanding of the various compaction mechanisms in the fungal kingdom. 


\section{Methods}

\section{Biological sample}

Peltaster fructicola strain LNHT1506 was obtained from a SBFS colony on the surface of a crabapple (Malus $\times$ micromalus Makino) fruit collected in Suizhong County, Liaoning Province, China. The cultures were purified by single spore isolation, maintained on potato dextrose agar (PDA) at $25^{\circ} \mathrm{C}$ and stored as glycerol stock (15\%) at $-80{ }^{\circ} \mathrm{C}$ in the Fungal Laboratory of Northwest A\&F University, Yangling, Shaanxi Province, China.

\section{DNA library preparation and Nanopore sequencing}

DNA was extracted using the Qiagen ${ }^{\curvearrowleft}$ Genomic DNA Kit following manufacturer guidelines (Cat\#13323, Qiagen). Quality and quantity of total DNA were evaluated using a NanoDrop $^{\text {Ts }}$ One UV-Vis spectrophotometer (Thermo Fisher Scientific, USA) and Qubit ${ }^{\bullet}$ 3.0 Fluorometer (Invitrogen, USA), respectively. The Blue Pippin system (Sage Science, USA) was used to retrieve large fragments by gel cutting. DNA repair was performed using a purchased DNA repair mix (NEBNext FFPE DNA Repair Mix, NEB M6630). End repair and dA-tailing used NEBNext End repair/dA-tailing Module (E7546, NEB). Ligation was then performed by Ligation Sequencing Kit 1D (SQK-LSK108, Oxford). MinION sequencing was performed as per manufacturer's guidelines using R9.4.1 flow cells (FLO-MIN106, ONT and controlled using Oxford Nanopore Technologies MinKNOW software. Flow cells were then transferred to Nanopore GridION × 5 (Oxford Nanopore Technologies, $\mathrm{UK})$ for nanopore single molecular sequencing.

\section{De novo genome assembly}

Canu v. 1.5 was used to assemble the Nanopore reads data set with default parameter [49]. One telomere was missing in a unitig by Canu assembly. To obtain a more complete assembly (telomere to telomere) the Nanopore data was analyzed along with Illumina data [12], and SPAdes v. 3.9.0 [50] was run with "-m 100 --nanopore nanopore.reads.fa --trusted-contigs canu.assembly.unitigs.fa -1Illumina.reads1.fastq -2 Illumina.reads1.fastq". We then obtained a chromosome-scale assembly with all 10 telomeres filled in at the ends of the five unitigs. According to unitig size from largest to smallest, we defined chromosome names as pf_ chr1 to pf_chr5. After the assembly step, we polished each set of unitigs with Pilon (v. 1.22) [51] using 256x of Illumina $2 \times 100$ bp paired-end reads, and then used software Nanopolish v. 0.10.1 (https:/github.com/jts/nanopolish) to do 'second polish' with nanopore fast 5 files. To achieve a high-quality assembly (more complete and without gaps) assembly, we conducted a 'third polish' stage by using Pilon.

\section{Gene and repeat annotation}

Gene annotation was accomplished using the BRAKER annotation pipeline to map expressed sequence tag evidence and ab initio gene predictions to the draft genome [52]. Filtered RNA-sequencing reads from a reference genome [12] and those sequenced in this study (GSE121872) were mapped to the genome with TopHat2 and putative transcripts were assembled with Cufflinks $[53,54]$. The putative transcripts were used in BRAKER v.2.1.0 as expressed sequence tag evidence. The genome completeness of assembly was assessed using BUSCO v. 1.2 [55]. In all cases, we ran BUSCO in the protein mode, using the Fungi reference database with '-l fungi -m OGS' parameter. Repeat sequences were identified by RepeatMasker v. 4.0.5 (http:// www.repeatmasker.org) and RepeatModeler v. 1.0.7 [56] pipeline with RepBase library (version: 20170127). The syntenic information was detected by MCScanX [57] and drawn by Circos as circular plots [58]. Transcribed coverage was computed by all transcribed exons divided by genome size. When multiple isoforms were found, the shortest length of isoform was chosen for further analysis. The length of intergenic and intron was extracted and calculated by the in house PERL scripts.

\section{Protein family analysis}

We identified ortholog pairs among selected fungal genomes by using OrthoMCL v. 2.0.9 (Table S10). To construct a genome-based phylogenic tree, MAFFT v. 7 was used to align single-copy ortholog pairs (http://mafft. cbrc.jp/alignment/server), conserved sites were extracted by using Gblocks v. 0.91b with the default parameters [59] and RAxML was used to construct maximum likelihood tree [60]. OrthoFinder was used to identify single or multi-copy genes from selected species [61].

\section{Transcriptome analysis}

We designed five treatments including mycelia on potato dextrose broth (PD) for $5 \mathrm{~d}$, mycelia on PD for $15 \mathrm{~d}$, mycelia on PD with PEG 6000 for 15 d, mycelia on potato dextrose agar (PDA) for $15 \mathrm{~d}$, and mycelia from artificial inoculations onto apple fruit in the field (15 d). Sequencing data from the latter two trials were published in our previous study [12].

For each sample, $10 \mu \mathrm{g}$ of total RNA were used for RNA-seq library construction. Before being used for directional RNA-seq library construction, oligo (dT)-conjugated magnetic beads (Invitrogen) was used to purify and concentrate Polyadenylated mRNAs. Purified mRNAs were then iron fragmented at $95^{\circ} \mathrm{C}$ and followed by end repair and 5'adaptor ligation. Next, reverse transcription was performed by using RT primer harboring 3' adaptor sequence and randomized hexamer. After cDNAs were purified and amplified, PCR products were purified corresponding to 200-500 bps, quantified and stored at $-80^{\circ} \mathrm{C}$ until used for sequencing. The libraries were paired-end sequenced with a read length of $125 \mathrm{bp}$ using Illumina $\mathrm{HiSeq}^{\mathrm{ms}} 2500$ 
sequencing platform. After filtration, clean reads were mapped to the reference genome assembled in this study using TopHat v. 2.0.9 with the parameter "-g 1" [62]. TopHat was used to conduct mapping our sequenced species including Peltaster fructicola, Ramichloridium luteum [13] and Colletotrichum fructicola [63]. For other species in this study, we used Hisat2 v. 2.1.0 to map reads to their genomes with the parameter "--dta-cufflinks --sra-acc <SRA accession number $>$ " [64]. SRA accession number was list in Table S11. A different gene expression profile was created using software Cufflinks v. 2.2.1 [53].

\section{Supplementary information}

Supplementary information accompanies this paper at https://doi.org/10. 1186/s12864-020-6732-8.

Additional file 1: Table S1. Genes in subtelomeric regions. 'L' means the left end of chromosomes. 'R' means the right end of chromosomes. Table S2. The annotations of collinear genes. Table S3. Repeat content in Peltaster fructicola. Table S4. Cluster containing unique gene in Peltaster fructicola but multi-genes in Zymoseptoria tritici. Table S5. Genes involved in carbohydrate metabolism. Table S6. Genes involved in amino acid metabolism. Table S7. Genes involved in nucleotide metabolism. Table S8. Genes involved in lipid metabolism. Table S9. Genes involved in cofactor metabolism. Table S10. Strain number and download source of selected species for phylogenomics. Table S11. Sequence Read Archive (SRA) accession number lists.

Additional file 2: Figure S1. Protein length distribution in Peltaster fructicola. Average length was 500 aa.

Additional file 3: Figure S2. Intron size distribution comparison. Box plot comparing the natural logarithm of intron size for the selected species and an outgroup member. Each box represents the interquartile range and outliers that are more than or less than 1.5 times the interquartile range are represented as dots in the boxplots.

Additional file 4: Figure S3. GO annotation information of Peltaster fructicola genome.

Additional file 5: Figure S4. Analysis of gene numbers and copies of Peltaster fructicola, Baudoinia compniacensis, Zymoseptoria tritici, Passalora fulva and Saccharomyces cerevisiae. Gene numbers of single copy and multi-copies are shown on the color bars.

\section{Abbreviations}

BLASTN: Basic Local Alignment Search Tool Nucleotide:

BUSCO: Benchmarking Universal Single Copy Orthologs; FPKM: Fragments Per Kilobase of transcript per Million fragments mapped; GO: Gene Ontology; SBFS: Sooty Blotch and Fly Speck

\section{Acknowledgements}

We would like to thank the anonymous reviewers for their kind and helpful comments on the original manuscript.

\section{Authors' contributions}

GS initiated and coordinated the project. BW, XL, GS and RZ conceived and planned the research. BW performed bioinformatics analyses. BW wrote the paper. GS, XL, MLG and TH revised the manuscript. All authors have read and approved the final version of the paper.

\section{Funding}

This work was supported by National Natural Science Foundation of China (31972220), and China Agriculture Research System (CARS-27). The funding bodies played no role in the design of the study and collection, analysis, and interpretation of data and in writing the manuscript.

\section{Availability of data and materials}

The genome assembly and gene annotation are available in the NCBI Genbank with WGS accession numbers of CP051139-CP051143. Raw sequencing reads are available in the NCBI Sequenced Read Archive under the accession numbers SRR11481813. The transcriptome data sets have been deposited at the Gene Expression Omnibus at the NCBI database under accession number GSE121872. The accession numbers obtained from NCBI database and subsequently analysed in this study are included in the Additional file 1 (Table S11).

Ethics approval and consent to participate

Peltaster fructicola strain LNHT1506 was obtained from a SBFS colony on the surface of a crabapple (Malus $\times$ micromalus Makino) fruit collected in Suizhong County, Liaoning Province, China. Collecting such samples is complied with institutional, national, and international guidelines and in accordance with local regulations.

Consent for publication

Not applicable.

\section{Competing interests}

The authors declare that they have no competing interests.

\section{Author details}

'State Key Laboratory of Crop Stress Biology in Arid Areas and College of Plant Protection, Northwest A\&F University, Yangling 712100, Shaanxi Province, China. ${ }^{2}$ MOE Key Laboratory for Intelligent Networks \& Network Security, Faculty of Electronic and Information Engineering, Xi'an Jiaotong University, Xi'an 710049, China. ${ }^{3}$ Department of Plant Pathology and Microbiology, lowa State University, Ames, IA 50011, USA. ${ }^{4}$ School of Environmental Sciences, University of Guelph, Guelph, Ontario N1G 2W1, Canada.

Received: 16 January 2020 Accepted: 14 April 2020

Published online: 23 April 2020

\section{References}

1. International Human Genome Sequencing Consortium. Initial sequencing and analysis of the human genome. Nature. 2001;409:860..

2. Djebali S, Davis CA, Merkel A, Dobin A, Lassmann T, Mortazavi, et al. Landscape of transcription in human cells. Nature. 2012;489:101.

3. Derelle E, Ferraz C, Rombauts S, Rouzé P, Worden AZ, Robbens $S$, et al. Genome analysis of the smallest free-living eukaryote Ostreococcus tauri unveils many unique features. Proc Natl Acad Sci U S A. 2006;103: 11647-52.

4. Brenner S, Elgar G, Sandford R, Macrae A, Venkatesh B, Aparicio S. Characterization of the pufferfish (Fugu) genome as a compact model vertebrate genome. Nature. 1993;366:265-8.

5. Venkatesh B, Gilligan P, Brenner S. Fugu: a compact vertebrate reference genome. FEBS Lett. 2000;476:3-7.

6. Goffeau A, Barrell BG, Bussey H, Davis RW, Dujon B, Feldmann H, et al. Life with 6000 genes. Science. 1996;274:563-7.

7. Cissé $\mathrm{OH}$, Pagni M, Hauser PM. De novo assembly of the Pneumocystis jirovecii genome from a single bronchoalveolar lavage fluid specimen from a patient. mBio. 2012:4:e00428-12.

8. Cissé $\mathrm{OH}$, Pagni M, Hauser PM, et al. Genome sequencing of the plant pathogen Taphrina deformans, the causal agent of peach leaf curl. mBio. 2013:4:e00055-13.

9. Ma L, Chen Z, Huang da W, Kutty G, Ishihara M, Wang H, et al. Genome analysis of three Pneumocystis species reveals adaptation mechanisms to life exclusively in mammalian hosts. Nat Commun. 2016;7:10740.

10. Gleason ML, Zhang R, Batzer JC, Sun G. Stealth pathogens: the sooty blotch and flyspeck fungal complex. Annu Rev Phytopathol. 2019;57:135-64.

11. Ismail SI, Batzer JC, Harrington TC, Crous PW, Lavrov DV, Li H, et al. Ancestral state reconstruction infers phytopathogenic origins of sooty blotch and flyspeck fungi on apple. Mycologia. 2016;108:292-302.

12. Xu C, Chen H, Gleason ML, Xu JR, Liu H, Zhang R, et al. Peltaster fructicola genome reveals evolution from an invasive phytopathogen to an ectophytic parasite. Sci Rep. 2016;6:22926.

13. Wang B, Liang X, Gleason ML, Zhang R, Sun G. Genome sequence of the ectophytic fungus Ramichloridium luteum reveals unique 
evolutionary adaptations to plant surface niche. BMC Genomics. 2017; 18:729.

14. Xu C, Zhang R, Sun G, Gleason ML. Comparative genome analysis reveals adaptation to the ectophytic lifestyle of sooty blotch and flyspeck fungi. Genome Biol Evol. 2017;9:3137-51.

15. Mohanta TK, Bae H. The diversity of fungal genome. Biol Proced Online. 2015;17:8.

16. Ohm RA, Feau N, Henrissat B, Schoch CL, Horwitz BA, Barry KW, et al. Diverse lifestyles and strategies of plant pathogenesis encoded in the genomes of eighteen Dothideomycetes fungi. PLoS Pathog. 2012;8: e1003037.

17. Kämper J, Kahmann R, Bölker M, Ma LJ, Brefort T, et al. Insights from the genome of the biotrophic fungal plant pathogen Ustilago maydis. Nature. 2006;444:97.

18. Blancq SML, Kase RS, Ploeg LHVD. Analysis of a Giardia lamblia rRNA encoding telomere with [TAGGG] $n$ as the telomere repeat. Nucleic Acids Res. 1991;19:5790.

19. Morrison HG, McArthur AG, Gillin FD, Aley SB, Adam RD, Olsen GJ, et al. Genomic minimalism in the early diverging intestinal parasite Giardia lamblia. Science. 2007;317:1921-6.

20. Louis EJ. The chromosome ends of Saccharomyces cerevisiae. Yeast. 1995;11: 1553-73.

21. van den Brink J, de Vries RP. Fungal enzyme sets for plant polysaccharide degradation. Appl Environ Microbiol. 2011;91:1477.

22. Goodwin SB, M'barek SB, Dhillon B, Wittenberg AH, Crane CF, Hane JK, et al. Finished genome of the fungal wheat pathogen Mycosphaerella graminicola reveals dispensome structure, chromosome plasticity, and stealth pathogenesis. PLoS Genet. 2011;7:781-8.

23. de Man TJ, Stajich JE, Kubicek CP, Teiling C, Chenthamara K, Atanasova L, et al. Small genome of the fungus Escovopsis weberi, a specialized disease agent of ant agriculture. Proc Natl Acad Sci U S A. 2016;113:3567.

24. Hane JK, Rouxel T, Howlett BJ, Kema GH, Goodwin SB, Oliver RP. A novel mode of chromosomal evolution peculiar to filamentous Ascomycete fungi. Genome Biol. 2011;12:R45.

25. Biemont C. Genome size evolution: within-species variation in genome size. Heredity (Edinb). 2008;101:297-8.

26. Prokopowich CD, Gregory TR, Crease TJ. The correlation between rDNA copy number and genome size in eukaryotes. Genome. 2003;46: 48-50.

27. Bergeron J, Drouin G. The evolution of $5 \mathrm{~S}$ ribosomal RNA genes linked to the rDNA units of fungal species. Curr Genet. 2008;54:123-31.

28. Gibbons JG, Branco AT, Yu S, Lemos B. Ribosomal DNA copy number is coupled with gene expression variation and mitochondrial abundance in humans. Nat Commun. 2014;5:4850.

29. Roller BR, Stoddard SF, Schmidt TM. Exploiting rRNA operon copy number to investigate bacterial reproductive strategies. Nat Microbiol. 2016;1:16160.

30. Goodenbour JM, Pan T. Diversity of tRNA genes in eukaryotes. Nucleic Acids Res. 2006;34:6137-46.

31. Wood V, Harris MA, McDowall MD, Rutherford K, Vaughan BW, Staines DM et al. PomBase: a comprehensive online resource for fission yeast. Nucleic Acids Res. 2012;40:D695-9.

32. Cridland JM, Macdonald SJ, Long AD, Thornton KR. Abundance and distribution of transposable elements in two Drosophila QTL mapping resources. Mol Biol Evol. 2013;30:2311-27.

33. Toome M, Ohm RA, Riley RW, Timothy YJ, Katherine LL, Bernard H, et al. Genome sequencing provides insight into the reproductive biology, nutritional mode and ploidy of the fern pathogen Mixia osmundae. New Phytol. 2014;202:554-64.

34. Katinka MD, Duprat $\mathrm{S}$, Cornillot E, Méténier $\mathrm{G}$, Thomarat F, Prensier $\mathrm{G}$, et al. Genome sequence and gene compaction of the eukaryote parasite Encephalitozoon cuniculi. Nature. 2001;414:450-45.

35. Nozaki H, Takano H, Misumi O, Terasawa K, Matsuzaki M, Maruyama S, et al. A 100\%-complete sequence reveals unusually simple genomic features in the hot-spring red alga Cyanidioschyzon merolae. BMC Biol. 2007;5:1-8.

36. Ibarra-Laclette E, Lyons E, Hernández-Guzmán G, Pérez-Torres CA, CarreteroPaulet $\mathrm{L}$, Chang TH, et al. Architecture and evolution of a minute plant genome. Nature. 2013;498:94-8.

37. Roest Crollius H, Jaillon O, Dasilva C, Ozouf-Costaz C, Fizames C, Fischer C, et al. Characterization and repeat analysis of the compact genome of the freshwater pufferfish Tetraodon nigroviridis. Genome Res. 2000;10:939-49.
38. Kelley JL, Peyton JT, Fiston-Lavier AS, Teets NM, Yee MC, Johnston JS, et al. Compact genome of the Antarctic midge is likely an adaptation to an extreme environment. Nat Commun. 2014;5:4611.

39. Amselem J, Lebrun $\mathrm{MH}$, Quesneville $\mathrm{H}$. Whole genome comparative analysis of transposable elements provides new insight into mechanisms of their inactivation in fungal genomes. BMC Genomics. 2015;16:141.

40. Böhme U, Otto TD, Cotton JA, Steinbiss S, Sanders M, Oyola SO, et al. Complete avian malaria parasite genomes reveal features associated with lineage specific evolution in birds and mammals. Genome Res. 2018;28:547-60

41. Ma J, Devos KM, Bennetzen JL. Analyses of LTR-retrotransposon structures reveal recent and rapid genomic DNA loss in rice. Genome Res. 2004;14: 860-9.

42. Hawkins JS, Proulx SR, Rapp RA, Wendel JF. Rapid DNA loss as a counterbalance to genome expansion through retrotransposon proliferation in plants. Proc Natl Acad Sci U S A. 2009;106:17811-6.

43. Dai $X$, Wang $H$, Zhou $H$, Wang L, Dvořák J, Bennetzen JL, et al. Birth and death of LTR-Retrotransposons in Aegilops tauschii. Genetics. 2018; 210:1039-51.

44. Giovannoni SJ, Thrash JC, Temperton B. Implications of streamlining theory for microbial ecology. ISME J. 2014;8:1553-65.

45. Stevenson BS, Schmidt TM. Life history implications of rRNA gene copy number in Escherichia coli. Appl Environ Microbiol. 2004;70:6670-7.

46. Kobayashi T. Regulation of ribosomal RNA gene copy number and its role in modulating genome integrity and evolutionary adaptability in yeast. Cell Mol Life Sci. 2011;68:1395-403.

47. Podlevsky JD, Bley CJ, Omana RV, Qi X, Chen JJ. The telomerase database. Nucleic Acids Res. 2007;36:D339-43.

48. Wieloch W. Chromosome visualisation in filamentous fungi. J Microbiol Meth. 2006:67:1-8

49. Koren S, Walenz BP, Berlin K, Miller JR, Bergman NH, Phillippy AM. Canu: scalable and accurate long-read assembly via adaptive k-mer weighting and repeat separation. Genome Res. 2017;27:722.

50. Bankevich A, Nurk S, Antipov D, Gurevich AA, Dvorkin M, Kulikov AS, et al. SPAdes: a new genome assembly algorithm and its applications to singlecell sequencing. J Comput Biol. 2012;19:455-77.

51. Walker BJ, Abeel T, Shea T, Priest M, Abouelliel A, Sakthikumar S, et al. Pilon: an integrated tool for comprehensive microbial variant detection and genome assembly improvement. PLoS One. 2014;9:e112963.

52. Hoff KJ, Lange S, Lomsadze A, Borodovsky M, Stanke M. Braker1: unsupervised RNA-seq-based genome annotation with Genemark-ET and Augustus. Bioinformatics. 2015;32:767-9.

53. Trapnell C, Williams BA, Pertea G, Mortazavi A, Kwan G, van Baren MJ, et al. Transcript assembly and quantification by RNA-Seq reveals unannotated transcripts and isoform switching during cell differentiation. Nat Biotechnol. 2010;28:511-55.

54. Kim D, Pertea G, Trapnell C, Pimentel H, Kelley R, Salzberg SL. TopHat2: accurate alignment of transcriptomes in the presence of insertions, deletions and gene fusions. Genome Biol. 2013;14:R36.

55. Simão FA, Waterhouse RM, loannidis P, Kriventseva EV, Zdobnov EM. BUSCO: assessing genome assembly and annotation completeness with single-copy orthologs. Bioinformatics. 2015;31:3210-2.

56. Saha S, Bridges S, Magbanua ZV, Peterson DG. Empirical comparison of abinitio repeat finding programs. Nucleic Acids Res. 2008;36:2284-94.

57. Wang Y, Tang H, Debarry JD, Tan X, Li J, Wang X, Lee TH, et al. MCScanX: a toolkitfor detection and evolutionary analysis of gene synteny and collinearity. Nucleic Acids Res. 2012;40:e49.

58. Krzywinski M, Schein Jl. Circos: an information aesthetic for comparative genomics. Genome Res. 2009;19:1639-45.

59. Castresama J. Selection of conserved blocks from multiple alignments for their use in phylogenetic analysis. Mol Biol Evol. 2000;17:540-52.

60. Stamatakis A. RAxML-VI-HPC: maximum likelihood-based phylogenetic analyses with thousands of taxa and mixed models. Bioinformatics. 2006;22: 2688-90.

61. Emms DM, Kelly S. OrthoFinder: solving fundamental biases in whole genome comparisons dramatically improves orthogroup inference accuracy. Genome Biol. 2015;16:157.

62. Trapnell C, Pachter L, Salzberg SL. TopHat: discovering splice junctions with RNA-Seq. Bioinformatics. 2009:25:1105-11. 
63. Liang X, Shang S, Dong $\mathrm{Q}$, Wang B, Zhang R, Gleason ML, et al. Transcriptomic analysis reveals candidate genes regulating development and host interactions of Colletotrichum fructicola. BMC Genomics. 2018;19:557.

64. Kim D, Langmead B, Salzberg SL. HISAT: a fast spliced aligner with low memory requirements. Nat Methods. 2015;12:357-36.

\section{Publisher's Note}

Springer Nature remains neutral with regard to jurisdictional claims in published maps and institutional affiliations.

Ready to submit your research? Choose BMC and benefit from:

- fast, convenient online submission

- thorough peer review by experienced researchers in your field

- rapid publication on acceptance

- support for research data, including large and complex data types

- gold Open Access which fosters wider collaboration and increased citations

- maximum visibility for your research: over $100 \mathrm{M}$ website views per year

At BMC, research is always in progress.

Learn more biomedcentral.com/submissions 